\section{Resistência do Mycobacterium tuberculosis às drogas em pacientes HIV+ em cinco municípios da Baixada Santista, São Paulo, Brasil}

\author{
Mycobacterium tuberculosis drug resistance in HIV \\ patients in Baixada Santista, São Paulo, Brazil
}

\author{
${ }^{1}$ Faculdade de Saúde \\ Pública, Universidade de \\ São Paulo, São Paulo, Brasil. \\ 2 Universidade Católica \\ de Santos, Santos, Brasil. \\ Correspondência \\ L. M. Rozman \\ Faculdade de Saúde Pública \\ Universidade de São Paulo. \\ Rua Torres Homem 503, \\ apto. 31, Santos, SP \\ 11025-021, Brasil. \\ lm.rozman@uol.com.br
}

\begin{abstract}
Since the early 1990s, an increase in Mycobacterium tuberculosis drug resistance has been reported, with high prevalence among HIV+ patients. We evaluated the sensitivity patterns of M. tuberculosis, resistance rate, and predisposing factors among HIV+ patients in Santos, São Vicente, Cubatão, Praia Grande, and Guarujá, São Paulo State, Brazil. The medical charts of 301 patients with positive cultures for $\mathrm{M}$. tuberculosis from 1993 to 2003 were reviewed. Resistance occurred in 57 patients (18.9\%), as follows: 32 (10.6\%) displayed multidrug-resistant tuberculosis (resistant to at least Rifampicin and Isoniazid); 4 (1.3\%) were resistant to two or more drugs; and 21 (7\%) were resistant to a single drug. Acquired resistance was observed in $70.1 \%$ of cases. Drug resistance was significantly associated with previous tuberculosis treatment, duration of HIV diagnosis, and previous hospitalization. In logistic regression analysis, only previous tuberculosis treatment adjusted by age remained as an independent risk factor $(\mathrm{OR}=$ 5.49; 95\%CI: 2.60-11.60). Drug resistance to at least one drug in 18.9\% and multidrug resistance in $10.6 \%$ of cases highlight the relevance of this problem in HIV patients in the Baixada Santista.
\end{abstract}

Tuberculosis; HIV; Drug Resistance
Luciana Martins Rozman 1

Augusto Hasiak Santo 1

Mauro Abrahão Rozman 2

\section{Introdução}

A tuberculose continua sendo um importante problema de saúde pública, especialmente em países em desenvolvimento 1. A Organização Mundial da Saúde (OMS) estima que entre 2002 e 2020 aproximadamente 1 bilhão de pessoas serão infectadas com o Mycobacterium tuberculosis, com mais de 150 milhões de doentes e 36 milhões de óbitos por tuberculose ${ }^{2}$. A incidência da doença está aumentando em várias regiões do mundo desde a década de 803 . O aumento no número de casos tem sido atribuído, sobretudo, à deterioração das medidas de controle, precárias condições econômicas e à epidemia da doença pelo HIV/AIDS 2,4,5.

O Brasil é o 15o colocado em número de casos estimados de tuberculose 6 , apresenta o maior número de casos da América Latina 7 e está entre os 22 países considerados prioritários pela OMS 8. O Estado de São Paulo apresenta o maior número absoluto de casos de tuberculose, com aproximadamente 21 mil notificações por ano. No Estado, os maiores coeficiente de incidência são encontrados na região da Baixada Santista 9 .

Além do aumento da incidência da tuberculose, desde 1988 tem ocorrido aumento no número de casos de resistência bacteriana às drogas antituberculose, inclusive nos países desenvolvidos, com alta prevalência entre os pacientes HIV+ 10 . $\mathrm{O}$ aumento do número de linhagens resistentes 
tem causado enorme preocupação, pois contribui para aumentar a proporção de mortes por tuberculose 11 , dificulta o tratamento e a prevenção da doença 7 .

O presente estudo objetiva avaliar a freqüência de resistência, o perfil de sensibilidade do $M$. tuberculosis às drogas antituberculose $\mathrm{e}$ os fatores predisponentes à resistência entre os indivíduos HIV+ nos municípios de Santos, São Vicente, Cubatão, Praia Grande e Guarujá no Estado de São Paulo.

\section{Material e métodos}

\section{População}

Foram selecionados todos os casos de tuberculose entre os pacientes HIV+ maiores de 13 anos de idade que apresentaram resultados de cultura positivos e cujas cepas isoladas e identificadas como M. tuberculosis foram submetidas a testes de sensibilidade pelo menos às seguintes drogas antituberculose: Isoniazida, Rifampicina, Pirazinamida, Etambutol e Estreptomicina. Esses casos foram selecionados no período de janeiro de 1993 a março de 2003 nas seguintes unidades de saúde: Centro de Referência em AIDS (Santos), Serviço de Atendimento de Especialidades (São Vicente), Núcleo de Vigilância à Saúde (Cubatão), Serviço de Atendimento de Especialidades (Praia Grande) e no Hospital-Dia William da Rocha (Guarujá).

Para obtenção dos dados laboratoriais, epidemiológicos e clínicos dos pacientes foi realizada pesquisa retrospectiva de seus prontuários por meio de formulário padronizado.

\section{Variáveis estudadas}

O desenho do estudo obedeceu aos princípios básicos de um estudo transversal, considerando a ocorrência de resistência como variável dependente e como variáveis independentes foram considerados:

- Sexo;

- Idade do paciente;

- Contagem de células CD4;

- Internação hospitalar prévia ao diagnóstico de tuberculose;

- Uso de anti-retrovirais ou de terapia anti-retroviral combinada potente (HAART: Highly Active Antiretroviral Therapy);

- Consumo de bebidas alcoólicas: foram registrados como positivos os casos em que, no prontuário, havia referência a consumo de bebidas alcoólicas, ainda que sem especificação da quantidade ou regularidade;
- Situação de moradia: foram considerados como positivos os casos em que constava no prontuário endereço domiciliar definido e foram considerados como negativos os casos em que constavam referências de ser o paciente "morador de rua";

- Uso de drogas injetáveis (UDI);

- Antecedentes de tratamento com drogas antituberculose.

\section{Definições}

Tuberculose resistente, tuberculose multirresistente, resistência secundária e resistência primária foram classificadas conforme critérios recomendados pela OMS 12:

- Tuberculose resistente: casos de tuberculose resistente a uma ou mais drogas;

- Tuberculose multirresistente: resistência a pelo menos Isoniazida e Rifampicina;

- Resistência primária: presença de resistência a uma ou mais drogas em pacientes que não haviam recebido medicamentos antituberculose anteriormente;

- Resistência secundária: presença de resistência a uma ou mais drogas em pacientes previamente tratados com drogas antituberculose, por um período mínimo de um mês;

- Resistência indeterminada: presença de resistência a uma ou mais drogas em pacientes sem informação em prontuário sobre tratamento anterior com drogas antituberculose.

\section{Análise estatística}

A avaliação inicial foi realizada por meio de análise univariada e pela análise estratificada utilizando os programas Epi Info 6.04 (Centers for Disease Control and Prevention, Atlanta, Estados Unidos) e STATA (Stata Corp., College Station, Estados Unidos). Para estimar o risco de desenvolver tuberculose resistente entre os pacientes $\mathrm{HIV}+$ foi calculado a odds ratio (OR) com intervalo de confiança de 95\% (IC95\%), considerando a existência ou não de tuberculose resistente como variável dependente e as várias exposições como variáveis independentes. Para análise multivariada, utilizou-se a regressão logística não condicional 13. Inicialmente foram incluídas no modelo todas as variáveis selecionadas na análise univariada e aquelas que não contribuíram para a explicação da presença da resistência foram excluídas, uma a uma (backward elimination) do modelo inicial. A significância estatística foi avaliada usando o teste de razão de verossimilhança comparando o "deviance" [-2ln(verossimilhança)] entre o modelo com e sem a variável. 
Este estudo foi aprovado pelo Comitê de Ética em Pesquisa da Faculdade de Saúde Pública, Universidade de São Paulo (Of.COEP/32/03) em 11 de fevereiro de 2003.

\section{Resultados}

Foram avaliados prontuários de 343 pacientes que no período de janeiro de 1993 a março de 2003 apresentaram culturas positivas cujas cepas foram isoladas e identificadas como M. tuberculosis com subseqüente avaliação da sensibilidade. Desses, 42 (12,2\%) foram excluídos do estudo, visto que em dois casos não foi encontrado resultado de exame anti-HIV, em seis casos não havia registro de consultas no prontuário e em 34 casos o prontuário não foi encontrado. Foram estudados, portanto, 301 casos, sendo a maioria do sexo masculino $(69,1 \%)$ com média de idade de 35,5 anos (desvio padrão de 8,19 anos), sendo a idade mínima 20 anos e a idade máxima 70 anos.

Dos casos analisados, $244(81,1 \%)$ foram sensíveis a todas as drogas testadas e $57(18,9 \%)$ foram resistentes ao menos a uma droga, sendo $32(10,6 \%)$ resistentes a no mínimo Isoniazida e Rifampicina (Tabela 1).

Entre os pacientes estudados, a resistência global (droga isolada e em associação) foi de: $8,6 \%$ (26 casos) para Rifampicina, $15,6 \%$ (47 ca- sos) para Isoniazida, 6,0\% (18 casos) para Pirazinamida, 2,6\% (8 casos) para Estreptomicina e $1,3 \%$ ( 4 casos) para Etambutol.

A Tabela 2 mostra a distribuição dos casos de tuberculose resistente segundo droga e resistência primária, secundária e indeterminada. Considerando-se o total desses casos, a resistência secundária foi observada em $40(70,2 \%)$ pacientes e resistência primária em 11 (19,3\%).

A análise estatística de potenciais fatores de risco associados à tuberculose resistente em pacientes HIV+ é mostrada na Tabela 3. Não houve associação estatisticamente significante entre as variáveis: sexo, situação de moradia, consumo de bebidas alcoólicas, uso de drogas injetáveis e uso de medicamentos anti-retrovirais ou HAART e tuberculose resistente. A variável tratamento anterior com drogas antituberculose mostrou a maior associação (OR = 4,93; IC95\%: 2,27-10,94), seguido pela tempo de evolução do HIV e hospitalização prévia ao diagnóstico de tuberculose.

Ajustando-se todas as variáveis testadas por faixa etária, o desenvolvimento de tuberculose resistente mostrou-se independentemente e de forma significativa associada à história de tratamento anterior com drogas antituberculose (OR = 5,49; IC95\%: 2,60-11,60). As variáveis: tempo de evolução do HIV e hospitalização prévia não mostraram associação significativa mesmo

Distribuição da população estudada segundo perfil de sensibilidade do Mycobacterium tuberculosis às drogas. Baixada Santista, São Paulo, Brasil, 1993-2003.

\begin{tabular}{|c|c|c|}
\hline Padrão de sensibilidade & $\mathbf{n}$ & $\%$ \\
\hline Sensíveis & 244 & 81,1 \\
\hline \multicolumn{3}{|l|}{ Resistentes } \\
\hline Rifampicina & 6 & 2,0 \\
\hline Isoniazida & 11 & 3,6 \\
\hline Pirazinamida & 1 & 0,3 \\
\hline Etambutol & 0 & 0,0 \\
\hline Estreptomicina & 3 & 1,0 \\
\hline Rifampicina + Isoniazida & 14 & 4,7 \\
\hline Rifampicina + Estreptomicina & 2 & 0,7 \\
\hline Rifampicina + Isoniazida + Pirazinamida & 12 & 4,0 \\
\hline Rifampicina + Isoniazida + Etambutol & 3 & 1,0 \\
\hline Rifampicina + Isoniazida + Estreptomicina & 1 & 0,3 \\
\hline Rifampicina + Isoniazida + Pirazinamida + Estreptomicina & 2 & 0,7 \\
\hline Isoniazida + Pirazinamida & 1 & 0,3 \\
\hline Isoniazida + Pirazinamida + Etambutol & 1 & 0,3 \\
\hline Subtotal & 57 & 18,9 \\
\hline Total & 301 & 100,0 \\
\hline
\end{tabular}


Distribuição dos casos de tuberculose resistente segundo padrão de resistência às drogas antituberculose. Baixada Santista, São Paulo, Brasil, $1993-2003$.

\begin{tabular}{|c|c|c|c|c|c|c|c|c|}
\hline \multirow[t]{2}{*}{ Perfil de resistência } & \multicolumn{2}{|c|}{$\begin{array}{c}\text { Resistência } \\
\text { primária }\end{array}$} & \multicolumn{2}{|c|}{$\begin{array}{c}\text { Resistência } \\
\text { adquirida }\end{array}$} & \multicolumn{2}{|c|}{$\begin{array}{c}\text { Resistência } \\
\text { indeterminada }\end{array}$} & \multicolumn{2}{|c|}{ Total } \\
\hline & $\mathrm{n}$ & $\%$ & $\mathbf{n}$ & $\%$ & $\mathbf{n}$ & $\%$ & $\mathbf{n}$ & $\%$ \\
\hline Rifampicina & 1 & 16,7 & 5 & 83,3 & 0 & 0,0 & 6 & 100,0 \\
\hline Isoniazida & 3 & 27,3 & 8 & 72,7 & 0 & 0,0 & 11 & 100,0 \\
\hline Pirazinamida & 0 & 0,0 & 1 & 100,0 & 0 & 0,0 & 1 & 100,0 \\
\hline Etambutol & 0 & 0,0 & 0 & 0,0 & 0 & 0,0 & 0 & 0,0 \\
\hline Estreptomicina & 2 & 66,7 & 0 & 0,0 & 1 & 33,3 & 3 & 100,0 \\
\hline Rifampicina + Isoniazida & 1 & 7,1 & 11 & 78,6 & 2 & 14,3 & 14 & 100,0 \\
\hline Rifampicina + Estreptomicina & 0 & 0,0 & 2 & 100,0 & 0 & 0,0 & 2 & 100,0 \\
\hline Rifampicina + Isoniazida + Pirazinamida & 4 & 33,3 & 7 & 58,3 & 1 & 8,4 & 12 & 100,0 \\
\hline Rifampicina + Isoniazida + Etambutol & 0 & 0,0 & 2 & 66,7 & 1 & 33,3 & 3 & 100,0 \\
\hline Rifampicina + Isoniazida + Estreptomicina & 0 & 0,0 & 1 & 100,0 & 0 & 0,0 & 1 & 100,0 \\
\hline Rifampicina + Isoniazida + Pirazinamida + Estreptomicina & 0 & 0,0 & 2 & 100,0 & 0 & 0,0 & 2 & 100,0 \\
\hline Isoniazida + Pirazinamida & 0 & 0,0 & 0 & 0,0 & 1 & 100,0 & 1 & 100,0 \\
\hline Isoniazida + Pirazinamida + Etambutol & 0 & 0,0 & 1 & 100,0 & 0 & 0,0 & 1 & 100,0 \\
\hline Total & 11 & 19,3 & 40 & 70,2 & 6 & 10,5 & 57 & 100,0 \\
\hline
\end{tabular}

quando ajustadas por faixa etária e foram excluídas do modelo final (Tabela 4).

\section{Discussão}

O presente estudo mostrou uma prevalência de $18,9 \%$ de resistência às drogas antituberculose nos pacientes HIV+ estudados. Estes dados não diferem muito dos relatados na literatura. Em estudo realizado com 431 pacientes com AIDS atendidos em Centro de Referência e Treinamento em DST/AIDS em São Paulo, encontrou-se resistência em 19\% dos casos 14. Em outro estudo, no Rio de Janeiro, a prevalência encontrada entre 127 casos atendidos em hospital de referência para AIDS foi de $16,6 \% 15$.

A taxa de resistência primária esperada para países em desenvolvimento é de $15 \%$ 16, enquanto a encontrada no presente estudo foi de 19,3\%. Esta freqüência foi também maior que a encontrada em outros inquéritos realizados no Brasil 17,18.

Ao contrário do que foi demonstrado em vários estudos 19,20, nesta casuística a freqüência de monorresistência a Rifampicina foi maior que a verificada para a Estreptomicina. A monorresistência adquirida a Rifampicina vem sendo referida como um aspecto peculiar dos casos de tuberculose/AIDS 21. Várias hipóteses foram aventadas para explicar este fenômeno sem que haja, entretanto, demonstração definitiva que fa- voreça algum aspecto em particular. Alguns autores relatam a não-adesão ao tratamento como a explicação mais plausível para estes casos 22 .

As maiores taxas encontradas de resistência global se relacionam às duas principais drogas do esquema de tratamento: Isoniazida $(15,6 \%)$ e Rifampicina (8,6\%). A multirresistência foi observada em $10,6 \%$ dos casos avaliados. Entre os pacientes HIV+ a multirresistência provoca aumento da mortalidade além de tratamento mais caro e tóxico 23 . Enquanto o tratamento com esquema básico de drogas custa US\$20,00, o tratamento para casos multirresistentes pode custar entre US $\$ 4.000,00$ e US $\$ 6.000,009$

O percentual de multirresistência verificado neste trabalho foi semelhante ao encontrado em estudos realizados com pacientes HIV+ no Brasil 14,15,24,25.

A taxa de resistência secundária aqui apresentada $(70,2 \%)$ foi maior que a registrada em outros estudos. Baptista et al. 26 encontraram taxa de $33,4 \%$; Pinto 14 , taxa de $45 \%$ e no estudo de Fandinho et al. 15, 13,3\% dos pacientes HIV+ foram tratados anteriormente com drogas antituberculose.

Alguns estudos têm sugerido que a infecção pelo HIV parece favorecer a resistência secundária 27,28. Várias hipóteses, como falhas no tratamento, reinfecção exógena 29 , interação medicamentosa com drogas para tratamento de AIDS 30 e má-absorção às drogas antituberculose, têm sido associados à resistência de M. tuber- 
Análise univariada para avaliação da associação entre tuberculose resistente e as variáveis de estudo.

Baixada Santista, São Paulo, Brasil, 1993-2003.

\begin{tabular}{|c|c|c|c|c|}
\hline Variável & $\begin{array}{l}\text { Sensível } \\
(n=244)\end{array}$ & $\begin{array}{c}\text { Resistente } \\
(n=57)\end{array}$ & Valor de $p$ & $\begin{array}{c}\text { OR } \\
\text { (IC95\%) }\end{array}$ \\
\hline \multicolumn{5}{|l|}{ Sexo } \\
\hline Masculino & 168 & 40 & 0,8457 & 1,00 \\
\hline Feminino & 76 & 17 & & $0,94(0,47-1,85)$ \\
\hline \multicolumn{5}{|c|}{ Faixa etária (anos) } \\
\hline $20-30$ & 62 & 20 & & 1,00 \\
\hline $31-34$ & 59 & 12 & 0,258 & $0,63(0,28-1,40)$ \\
\hline $35-49$ & 52 & 14 & 0,648 & $0,83(0,38-1,81)$ \\
\hline $50-70$ & 71 & 11 & 0,076 & $0,48(0,21-1,08)$ \\
\hline \multicolumn{5}{|l|}{ Domicílio } \\
\hline Não & 30 & 5 & 0,4550 & 1,00 \\
\hline Sim & 214 & 52 & & $1,46(0,50-4,55)$ \\
\hline \multicolumn{5}{|c|}{ Consumo de álcool } \\
\hline Não & 50 & 11 & 0,4997 & 1,00 \\
\hline Sim & 83 & 24 & & $1,31(0,55-3,17)$ \\
\hline \multicolumn{5}{|c|}{ Uso de droga injetável } \\
\hline Não & 138 & 29 & 0,3113 & 1,00 \\
\hline Sim & 95 & 27 & & $1,35(0,72-2,54)$ \\
\hline \multicolumn{5}{|c|}{ Uso de anti-retrovirais } \\
\hline Não & 195 & 42 & 0,2640 & 1,00 \\
\hline Sim & 44 & 14 & & $1,48(0,70-3,11)$ \\
\hline \multicolumn{5}{|l|}{ HAART } \\
\hline Não & 178 & 40 & 0,6401 & 1,00 \\
\hline Sim & 61 & 16 & & $1,17(0,58-2,35)$ \\
\hline \multicolumn{5}{|c|}{ Hospitalização prévia } \\
\hline Não & 168 & 33 & 0,0497 & 1,00 \\
\hline Sim & 51 & 19 & & $1,90(0,94-3,82)$ \\
\hline \multicolumn{5}{|c|}{ Tempo de evolução do HIV (anos) } \\
\hline Até 4 & 176 & 31 & 0,01258 & 1,00 \\
\hline 5 ou mais & 67 & 25 & & $2,12(1,11-4,04)$ \\
\hline \multicolumn{5}{|c|}{ Linfócitos CD4+ (por mm³) } \\
\hline 500 ou mais & 21 & 22 & & 1,00 \\
\hline $200-499$ & 55 & 19 & 0,510 & $1,45(0,81-3,27)$ \\
\hline $0-199$ & 104 & 22 & 0,830 & $0,88(0,30-2,61)$ \\
\hline \multicolumn{5}{|c|}{ Tratamento anterior } \\
\hline Não & 118 & 11 & 0,0000 & 1,00 \\
\hline Sim & 87 & 40 & & $4,93(2,27-10,94)$ \\
\hline
\end{tabular}

HAART: Highly Active Antiretroviral Therapy.

culosis durante ou logo após o tratamento em pacientes $\mathrm{HIV}+31$.

Embora a taxa de resistência secundária aqui apresentada tenha sido alta não foi possível detectar associação entre resistência e fatores considerados predisponentes ao abandono do tratamento tais como uso de drogas injetáveis, alcoolismo e falta de moradia.

A internação anterior mostrou associação com a resistência em análise univariada, no en- tanto, essa associação não foi confirmada em análise multivariada. A qualidade da informação dos prontuários e inexistência de informação a respeito do tempo de hospitalização, duração e tipo de isolamento e outros parâmetros especificamente vinculados à estrutura hospitalar e à assistência em cada local dificultaram uma análise pormenorizada e mais adequada. 
Tabela 4

Análise multivariada segundo variáveis explicativas da resistência às drogas antituberculose, ajustada por faixa etária. Baixada Santista, São Paulo, Brasil, 1993-2003.

\begin{tabular}{lcc}
\hline Variável & OR & IC95\% \\
\hline Tratamento anterior & & \\
Não & 1,00 & \\
Sim & 5,49 & $2,60-11,60$ \\
Faixa etária (anos) & & \\
$20-30$ & 1,00 & $0,18-1,15$ \\
$31-34$ & 0,46 & $0,20-1,22$ \\
$35-49$ & 0,50 & $0,11-0,75$ \\
$50-70$ & 0,29 & \\
\hline
\end{tabular}

A contagem de células CD4+ em pacientes HIV+ tem sido considerada um bom marcador para progressão da doença, uma vez que existe uma correlação inversa entre contagem de CD4 e desenvolvimento de infecções oportunistas e óbito 32,33 . Estudos em países desenvolvidos têm demonstrado que, embora a tuberculose seja diagnosticada em pacientes HIV+ com diferentes contagens de $\mathrm{CD} 4+$, a incidência de tuberculose é maior naqueles com contagem de linfócitos abaixo de $200 / \mathrm{mm}^{3} 34,35$. Neste estudo não houve associação entre resistência e contagem de CD4+, resultado concordante com o encontrado na literatura 36 .

Estudos recentes têm demonstrado redução da incidência de doenças oportunistas, incluindo a tuberculose, após a introdução de HAART 37,38. No entanto, não foi possível analisar o efeito protetor de HAART para o desenvolvimento de tuberculose resistente. O curto período de seguimento de pacientes que receberam a medicação e o relativamente pequeno número de pacientes tratados com HAART podem ter influenciado na análise de tal associação.

O maior tempo de diagnóstico de HIV esteve associado à tuberculose resistente em análise univariada, porém a variável não se mostrou estatisticamente associada em análise multivariada.

A única variável que se mostrou independentemente associada à tuberculose resistente em $\mathrm{HIV}+$ foi tratamento anterior com drogas antituberculose, resultado concordante com a literatura 20,39 .

A importância da co-infecção pelo HIV na determinação da resistência deve ser analisada pela comparação com a ocorrência de resistência em soronegativos. Diversos estudos têm demonstrado que a co-infecção do M. tuberculosis com o
HIV é um fator de risco independentemente associado à tuberculose resistente $40,41,42,43$. Outros autores não encontraram associação entre HIV e resistência às drogas antituberculose 19,20,44,45,46.

Os estudos que mostraram associação foram realizados em locais em que as taxas de resistência eram crescentes 41,42 . Segundo os autores, essa associação pode ser entendida com base nos conhecimentos da história natural da tuberculose. O intervalo entre a infecção e o desenvolvimento da doença nos indivíduos imunocompetentes pode ser de anos ou décadas; os casos atuais de tuberculose nesses indivíduos refletem o padrão de resistência de um período em que estas taxas eram baixas, anos ou décadas antes do aparecimento da doença ativa. Nos indivíduos HIV+, a progressão da infecção para doença é muito mais rápida e os casos atuais refletem um padrão de resistência mais recente, com taxas maiores. Os padrões de resistência dos indivíduos HIV+ refletiriam as tendências recentes na comunidade 42,47. Outras hipóteses foram apresentadas para explicar uma maior ocorrência de casos resistentes em indivíduos HIV+: (a) as cepas resistentes se mostraram, in vitro, menos viáveis que as sensíveis e menos virulentas; cepas que somente seriam capazes de provocar doença em indivíduos imunodeprimidos; (b) podem existir fatores de risco comuns para co-infecção e resistência às drogas antituberculose, como uso de drogas injetáveis e hospitalização; (c) o tratamento de pacientes imunodeprimidos pode falhar porque esses pacientes possuem maior número de bacilos e podem ter maior diversidade genética dos microorganismos; (d) os indivíduos co-infectados podem estar sujeitos mais freqüentemente a monoterapia funcional. Enquanto a replicação bacteriana é suprimida em pacientes HIV negativos logo após os dois primeiros meses de tratamento com três drogas, o mesmo pode não acontecer nos indivíduos HIV+. Em função da meia-vida mais curta da Isoniazida o bacilo pode ficar exposto apenas à Rifampicina, já que na segunda fase o esquema de tratamento é composto especificamente por essas duas drogas 23 .

Ainda que alguns trabalhos indiquem elevada proporção de resistência às drogas antituberculose em indivíduos HIV negativos 25,48, a Vigilância de Resistência às Drogas Antituberculose da Secretaria de Estado da Saúde de São Paulo mostra prevalências de resistência às drogas, no total de indivíduos com tuberculose, muito inferiores às encontradas nos estudos realizados em indivíduos HIV+: a resistência global a qualquer droga e a multirresistência foram, respectivamente, de 1,3 e 9\% para o Brasil, 2,4 e 6,4\% para o interior do Estado de São Paulo, 3,9 e 14\% para o litoral e 3,1 e 9,5\% para a Grande São Paulo ${ }^{9}$. 
Não existem informações sobre a evolução temporal da resistência na Baixada Santista. Caso se confirme a maior prevalência de resistência em indivíduos HIV+, a tuberculose nesse grupo deve contribuir para o aumento da proporção de tuberculose resistente na Baixada Santista e em outras regiões.
Considerando a elevada proporção de resistência secundária na região entre os pacientes HIV+ encontrada nesse estudo, é de fundamental importância a intensificação das ações que visam à redução do abandono de tratamento nos doentes com co-infecção.

\section{Resumo}

No início dos anos 90, observou-se aumento da resistência do Mycobacterium tuberculosis às drogas antituberculose, com alta prevalência entre pacientes $H I V+$. Neste estudo, foram avaliados a freqüência de resistência, o perfil de sensibilidade do $\mathrm{M}$. tuberculosis às drogas e os fatores predisponentes à resistência entre indivíduos HIV+ nos municípios de Santos, São Vicente, Cubatão, Praia Grande e Guarujá, Estado de São Paulo, Brasil. Foram pesquisados os prontuários de 301 pacientes com resultado de cultura positivo entre 1993 e 2003. A resistência ocorreu em 57 (18,9\%) pacientes com a seguinte distribuição: 32 (10,6\%) apresentaram tuberculose multirresistente (resistência ao menos à Rifampicina e Isoniazida); 4 (1,3\%) casos apresentaram resistência a duas ou mais drogas e 21 (7\%) à monorresistência. Observou-se resistência secundária em 70,2\% dos casos. Estatisticamente associadas à tuberculose resistente foram: tratamento anterior com drogas antituberculose, tempo de diagnóstico de HIV e hospitalização prévia. Em análise multivariada, apenas tratamento anterior, ajustado por faixa etária, mostrou-se associado (OR = 5,49; IC95\%: 2,60-11,60). A ocorrência de resistência em 18,9\% dos casos e multirresistência em aproximadamente 10\% confirmam a relevância deste problema entre pacientes $\mathrm{HIV}+n a$ Baixada Santista.

Tuberculose; HIV; Resistência a Drogas

\section{Colaboradores}

L. M. Rozman redigiu o artigo. A. H. Santo realizou a revisão de todo o artigo. M. A. Rozman auxiliou na análise estatística dos dados e nas respostas aos pareceres. 


\section{Referências}

1. Fundação Nacional de Saúde. Guia de vigilância epidemiológica. 5a Ed. Brasília: Fundação Nacional de Saúde; 2002.

2. World Health Organization. Tuberculosis. Geneva: World Health Organization; 2002. (Fact Sheet, 104).

3. Narain JP, Raviglione MC, Kochi A. HIV-associated tuberculosis in developing countries; epidemiology and strategies for prevention. Tuber Lung Dis 1992; 73:311-21.

4. Catwell MF, Snider DE, Cauthen GM, Onorato IM Epidemiology of tuberculosis in the United States, 1985 through 1992. JAMA 1994; 272:535-9.

5. García-García MR, Valdespino-Gómez JL, GarcíaSancho MC, Salcedo-Álvarez RA, Zacarias F, Sepulveda-Amor JS. Epidemiology of AIDS and tuberculosis. Bull Pan Am Health Organ 1995; 29:37-58.

6. World Health Organization. Global tuberculosis control: WHO report. Geneva: World Health Organization; 2003.

7. Rossetti MLR, Valim ARM, Silva MSN, Rodrigues VS. Tuberculose resistente: revisão molecular. Rev Saúde Pública 2002; 36:525-32.

8. Natal S, Valente JG, Sánchez AR, Penna MLR. Resistência a isoniazida e rifampicina e história de tratamento anterior para tuberculose. Cad Saúde Pública 2003; 19:1277-81.

9. Centro de Vigilância Epidemiológica, Secretaria de Estado da Saúde. A tuberculose no Estado de São Paulo. http://www.cve.saude.sp.gov.br (acessado em 24/Out/2006).

10. Schwoebel V, Dicludt B, Binoist A, Haeghebaert S, Torrea G, Vincent V, et al. Multidrug resistant tuberculosis in France 1992-4: two case-control studies. BMJ 1998; 317:630-1.

11. Snider DE, Roper WL. The new tuberculosis. N Engl J Med 1992; 32:106-10.

12. Vareldzis BP, Grosset J, Kantos I, Crofton J, Laszlo A, Felten M, et al. Drug-resistant tuberculosis: laboratory issues. Tuber Lung Dis 1994; 75:1-7.

13. Hosmer JR, Lemeshow S. Applied logistic regression. New York: John Wiley and Sons; 1989.

14. Pinto WP. Tuberculose e resistência a drogas em pacientes atendidos em um centro de referência para síndrome da imunodeficiência adquirida em São Paulo - Brasil [Tese de Doutorado]. São Paulo: Faculdade de Medicina, Universidade de São Paulo; 1998.

15. Fandinho FCO, Kritski AL, Hofer C, Conde Jr. H, Ferreira RMC, Silva MG, et al. Drug resistance patterns among hospitalized tuberculous patients in Rio de Janeiro, Brazil, 1993-1994. Mem Inst Oswaldo Cruz 1999; 94:543-7.

16. Toman K. Tuberculosis, detección de casos y quimioterapia. Preguntas y respuestas. Washington DC: Organización Panamericana de la Salud; 1980. (Publicación Científica, 392).

17. Barreto AM, Martins FM. Estudo da resistência primária no Brasil no período de 1986 a 1988. Bol Campanha Nac Tuberc 1988; 2:21-5.

18. Silva EAM, Sato DN, Telles MAS, Martins MC, Palaci M, Ueki SYM. Perfil de resistência de Mycobacterium tuberculosis no Estado de São Paulo, 1986 a 1990. Rev Inst Adolfo Lutz 1992; 52:37-40.
19. Cohn DL, Bustreo F, Raviglione MC. Drug-resistant tuberculosis: review of the worldwide situation and the WHO/IUATLD Global Surveillance Project. Clin Infect Dis 1997; 24 Suppl 1:121-30.

20. Espinal MA, Laserson K, Camacho M, Fuscheng Z, Kim SJ, Tladi RE, et al. Determinants of drug-resistant tuberculosis: analysis of 11 countries. Int J Tuberc Lung Dis 2001; 5:885-6.

21. Sandman L, Schluger NW, Davidow AL, Bonk S. Risk factors for rifampin-monoresistant tuberculosis: a case-control study. Am J Respir Crit Care Med 1999; 159:468-72.

22. Munsiff SS, Joseph S, Ebrahimzadeh A, Frieden TR. Rifampin-monoresistant tuberculosis in New York city, 1993-1994. Clin Infect Dis 1997; 25:1465-7.

23. Dye C, Watt, CJ, Bleed D. Low access to a highly effective therapy: a challenge for international tuberculosis control. Bull World Health Organ 2002; 80:437-44.

24. Machado ES, Cruz ML, Souza MJ, Ribeiro S, Silva MG, Giordani ML, et al. Drug-resistant tuberculosis in HIV+ and HIV- patients. In: International Conference on AIDS, 10; International Conference on STD, 5. Abstracts. Yokohama: International AIDS Society; 1994. p. 168.

25. Almeida-da-Silva PE, Osório M, Reinhardt MC, Fonseca LS, Delladostin AO. Drug resistance of strains of Mycobacterium tuberculosis isolated in Brazil. Microbes Infect 2001; 3:1111-3.

26. Baptista IMFD, Oelemann MC, Opromolla DVA, Suffys PN. Drug resistance and genotypes of strains of Mycobacterium tuberculosis isolated from human immunodeficiency virus-infected and noninfected tuberculosis patients in Bauru, São Paulo, Brazil. Mem Inst Oswaldo Cruz 2002; 97:1147-52.

27. March F, Garriga X, Rodríguez P, Moreno C, Garrigó $\mathrm{M}$, Coll P, et al. Acquired drug resistance in $M y-$ cobacterium tuberculosis isolates recovered from compliant patients with human immunodeficiency virus - associated tuberculosis. Clin Infect Dis 1997; 25:1044-7.

28. Bradford WZ, Martin JN, Reingold AL, Schecter GF Hopewell PC, Small PM. The changing epidemiology of acquired drug-resistant tuberculosis in San Francisco, USA. Lancet 1996; 348:928-31.

29. Small PM, Shafer RW, Hopewell PC. Exogenus reinfection with drug-resistant Mycobaterium tuberculosis in patients with advanced HIV infection. N Engl J Med 1993; 328:1137-44.

30. Lee BL, Safrin S. Interactions and toxicities of drugs used in patients with AIDS. Clin Infect Dis 1992; 14:773-9.

31. Peloquim CA, MacPhee AA, Berning SE. Malabsorption of antimycobacterial medications. $\mathrm{N}$ Engl J Med 1993; 329:1122-3.

32. Nunn P, Elliott AM, McAdam KP. Impact of human immunodeficiency virus on tuberculosis on developing countries. Thorax 1994; 49:511-8.

33. Ackah A, Coulibaly D, Digbeu H, Diallo K, Vetter K, Coulibaly I, et al. Response to treatment, mortality, and CD4 lymphocyte counts in HIV-infected persons with tuberculosis in Abidjan, Côte d'Ivoire. Lancet 1995; 345:607-10. 
34. Di Perri G, Vento S, Cruciani M, Micciolo R, Concia E, Bassetti D. Tuberculosis and HIV infection. N Engl J Med 1991; 325:1882-3.

35. Antonucci G, Girardi E, Raviglione M, Ippolito G. Risk factors for tuberculosis in HIV persons. A prospective cohort study. JAMA 1995; 274:143-8.

36. Murray J, Sonnenberg P, Shearer S, GodfreyFaussett P. Drug-resistant pulmonary tuberculosis in a cohort of southern African goldminers with a high prevalence of HIV infection. S Afr Med J 2000; 90:381-6.

37. Badri M, Wilson D, Wood R. Effect of highly active antiretroviral therapy on incidence of tuberculosis in South Africa: a cohort study. Lancet 2002; 359:2059-64

38. Santoro-Lopes G, Pinho AMF, Harrison LH, Schechter M. Reduced risk of tuberculosis among Brazilian patients with advanced human immunodeficiency virus infection treated with highly active antiretroviral therapy. Clin Infect Dis 2002; 34:543-6.

39. World Health Organization/International Union Against Tuberculosis and Lung Disease. Global project on anti-tuberculous drug resistance surveillance. Report 2: prevalence and trends. Geneva: World Health Organization; 2000.

40. Frieden TR, Sterling T, Mendez AP, Kilburn JO, Cauthen GM, Dooley SW. The emergence of drug-resistant tuberculosis in New York City. N Engl J Med 1993; 328:521-6.
41. Salomon N, Perlman DC, DePalo VA, Kolokathis A, Wilets I. Drug-resistant tuberculosis: factors associated with rise in resistance in an HIV-infected urban population. Mt Sinai J Med 1994; 61:341-8.

42. Gordin FM, Nelson ET, Matts JP, Cohn PL, Ernest J, Benator D, et al. The impact of human immunodeficiency virus infection on drug- resistant tuberculosis. Am J Respir Crit Care Med 1996; 154:1478-83.

43. Liu Z, Shilkret KL, Finelli L. Epidemiology of drugresistant tuberculosis in New Jersey from 1991 to 1995. Int J Epidemiol 1998; 27:121-6.

44. Wilkinson D, Pillay M, Davies G, Sturn A. Resistance to antituberculosis drugs in rural South Africa: rates, patterns, risks, and transmission dynamics. Trans R Soc Trop Med Hyg 1996; 90:692-5.

45. Boudville I, Wong S, Snodgrass I. Drug resistant tuberculosis in Singapore. Ann Acad Med Singapore 1997; 26:549-56.

46. Barroso EC, Mota RMS, Santos RO, Sousa ALO, Barroso JB, Rodrigues JLN. Fatores de risco para tuberculose multirresistente adquirida. J Pneumol 2003; 29:89-97.

47. Kochi A, Vareldzis B, Styblo K. Multidrug-resistant tuberculosis and its control. Res Microbiol 1993; 144:104-10.

48. Barroso EC, Rodrigues JLN, Pinheiro VGF, Campelo CL. Prevalência da tuberculose multirresistente no Estado do Ceará, 1990-1999. J Pneumol 2001; 27:310-4.

Recebido em 14/Out/2005

Versão final reapresentada em 31/Out/2005

Aprovado em 18/Dez/2006 